\title{
O Uso do Moodle como Reforço ao Ensino Presencial de Parasitologia e Micologia no Curso de Graduação em Medicina
}

\author{
The Moodle as a Reinforcement to Classroom \\ Teaching of Course Parasitology and \\ Mycology in Undergraduate Medical Course
}

Adelina Mezzari Isabele Iser ${ }^{I I}$ Adília Maria Pereira Wiebbelling ${ }^{I I}$ Liane Tarouco

\section{PALAVRAS-CHAVE \\ - Ensino Presencial. \\ - Aprendizagem. \\ - Educação Médica.}

\section{KEYWORDS}

- Classroom Teaching.

- Learning.

- Medical Education.

Recebido em: 04/06/2012

Aprovado em: 02/10/2012

REVISTA BRASLLEIRA DE EDUCAÇÃO MÉDICA
' Universidade Federal do Rio Grande do Sul, Rio Grande do Sul, RG, Brasil.

"Universidade Federal de Ciências da Saúde de Porto Alegre, Rio Grande do Sul, RG, Brasil. 


\section{INTRODUÇÃO}

O ensino e a aprendizagem, atualmente, envolvem ação, reflexão e estratégias de ensino por parte do professor. Muitos docentes já perceberam que precisam lançar mão de técnicas que aumentem a participação e a aprendizagem do aluno ${ }^{1}$.

Às vezes, as práticas pedagógicas utilizadas no ensino da graduação são questionadas, tendo como consequência mudanças no ensino, como as centradas no aluno e na resolução de problemas ${ }^{2}$. Castanho ${ }^{3}$, em estudo com professores do ensino superior da área da saúde, perguntou sobre a prática pedagógica cotidiana destes, usando a metodologia da história oral temática, e concluiu que não é possível prescindir de formação pedagógica para o exercício do magistério superior e que é fundamental introduzir inovações que atinjam as bases estruturais do ensino.

Os ambientes virtuais de aprendizagem (AVA) são sistemas que possibilitam a implantação de técnicas cuja finalidade é o ensino-aprendizagem ${ }^{4}$. Existem plataformas que permitem a utilização de mecanismos para complementar o ensino. Uma delas é o Modular Object Oriented Distance Learning (Moodle), um sistema de gestão de conteúdos, em código de fonte aberta (http://www.moodle.org), utilizado na educação como plataforma de gestão do ensino e aprendizagem. $\mathrm{O}$ emprego dessa ferramenta possibilita aplicar o ensino também fora da sala de aula. Permite a interação entre o professor e o aluno na entrega e correção de trabalhos, por meio de chats e fóruns, além de disponibilizar tarefas para serem corrigidas neste mesmo ambiente posteriormente ${ }^{5,6}$. Esta plataforma tem sido usada por professores em todas as áreas de ensino, inclusive na da saúde.

Ao inserir estratégias de melhorias no ensino tendo como objetivo desenvolver habilidades e competências nos alunos, o professor precisará, ao longo deste período, manter uma avaliação contínua do aluno. O Moodle, sendo um sistema de gerenciamento do ensino a distância, auxilia o professor a complementar suas técnicas de ensino presencial. Disponibiliza para o professor um ambiente para suas aulas, um link na internet, exercícios, uma pesquisa de opinião, entre outros. Este sistema pode ser aplicado no início, durante e/ou no final da disciplina, como ferramenta de avaliação ${ }^{7}$.

Na concepção construtivista da aprendizagem, o aprender não necessita reproduzir a realidade, mas deve ser capaz de elaborar uma representação pessoal sobre esta e seus conteúdos. Sendo assim, o aluno, ao se defrontar com problemas simulados, tem a oportunidade de aprender fazendo. Quando ele erra e aprende com o próprio erro, constrói seu aprendizado por meio desta identificação. Neste caso, o professor acompanha o desenvolvimento do aluno, atuando como avaliador, facilitador ou consultor. Esta estratégia pedagógica constitui um instrumento de desenvolvimento das competências e habilidades na área da saúde ${ }^{8,9}$.

Este estudo tem por objetivo acompanhar o desenvolvimento das mudanças que ocorreram desde março de 2009 na disciplina de Parasitologia e Micologia Médica do curso de Medicina da Universidade Federal de Ciências da Saúde de Porto Alegre (UFCSPA), a partir de sua inserção no ambiente Moodle institucional. A disciplina ocorre no primeiro ano do curso, sendo que a metade dos alunos assistem no primeiro semestre, e a outra metade, no segundo. Foi escolhido o Moodle como suporte para a elaboração desta mudança porque favorece a interação e a construção conjunta do conhecimento, sendo esta uma forma de aprendizagem.

\section{METODOLOGIA}

O aluno do curso de Medicina da Universidade Federal de Ciências da Saúde de Porto Alegre (UFCSPA) matriculado na disciplina de Parasitologia e Micologia Médica, na primeira série, recebe como informação uma dinâmica interdisciplinar que vem ocorrendo desde março de 2009. A disciplina, até então totalmente presencial, passou a desenvolver atividades de ensino em sua página, utilizando ferramentas no ambiente Moodle institucional. Os alunos são inseridos neste ambiente, onde são preparadas atividades interativas e os conteúdos relacionados a cada aula presencial, antes que elas ocorram.

Nestas atividades estão incluídos estudo de casos clínicos, questionários de verdadeiro ou falso, questões de múltipla escolha, cruzadinhas, associação de colunas e questões dissertativas. A resolução dos estudos de casos clínicos tem como finalidade estimular o raciocínio clínico, aliando situações do cotidiano ao conteúdo teórico, e culminar no diagnóstico. Nos questionários de verdadeiro ou falso, quando o enunciado está incorreto, o aluno recebe imediatamente um feedback com as devidas correções. As questões de escolha simples ou múltipla se assemelham ao modelo de provas objetivas. As cruzadinhas buscam estimular o aprendizado proporcionando uma atividade lúdica. A atividade de associar colunas é realizada com o intuito de firmar conceitos básicos da disciplina. As questões dissertativas induzem a compreensão do aluno em relação ao conteúdo e estimulam o aprimoramento das habilidades de expressão do acadêmico. Os alunos recebem feedback de correção em todas as atividades disponibilizadas e têm acesso ao monitor tutor para a resolução de dúvidas.

Todas as atividades são disponibilizadas na página da disciplina no Moodle pelo menos sete dias antes de cada avaliação. Deste modo, ao fazer os exercícios, o aluno tem um período para realizar a revisão do conteúdo da prova até a vés- 
pera da avaliação. Cada atividade é pontuada, e a nota faz parte da média final da disciplina.

Também é disponibilizado aos alunos no ambiente Moodle um atlas de Micologia e de Parasitologia com figuras de micoses, fungos e parasitos vistos em aula teórica e reforçados nas aulas práticas. As avaliações do aprendizado são feitas ao longo do semestre tanto na forma tradicional quanto por meio das atividades desenvolvidas no ambiente Moodle. Nesta modalidade se avalia a frequência de acesso pelos alunos, sua participação na disciplina e as soluções dos estudos de casos clínicos ou exercícios de fixação dos conteúdos. Este acompanhamento resulta em nota.

Para a constante avaliação desta modalidade de ensino na disciplina, os alunos são convidados a responder, sem se identificar, a um questionário sobre o uso da ferramenta Moodle nas atividades disponibilizadas e verificar seu conhecimento quanto à utilização desta metodologia em relação ao ensino presencial tradicional. O gerenciamento destas atividades da disciplina e das disponibilizadas no Moodle é realizado pelo monitor tutor e supervisionado pelos professores.

\section{RESULTADOS}

Para ter acesso às atividades no Moodle através das ferramentas deste sistema, os alunos matriculados na disciplina podem acessar o site com seu número de usuário e senha registrados na universidade. A página visualizada contém a tela de apresentação da disciplina e os links na forma de tópicos, como descritos na Figura 1. As atividades apresentadas aos alunos, o material didático e os estudos de casos clínicos para serem desenvolvidos são mostrados na Figura 2.

FigURA 1

Tela de apresentação da disciplina e os links em forma de tópicos

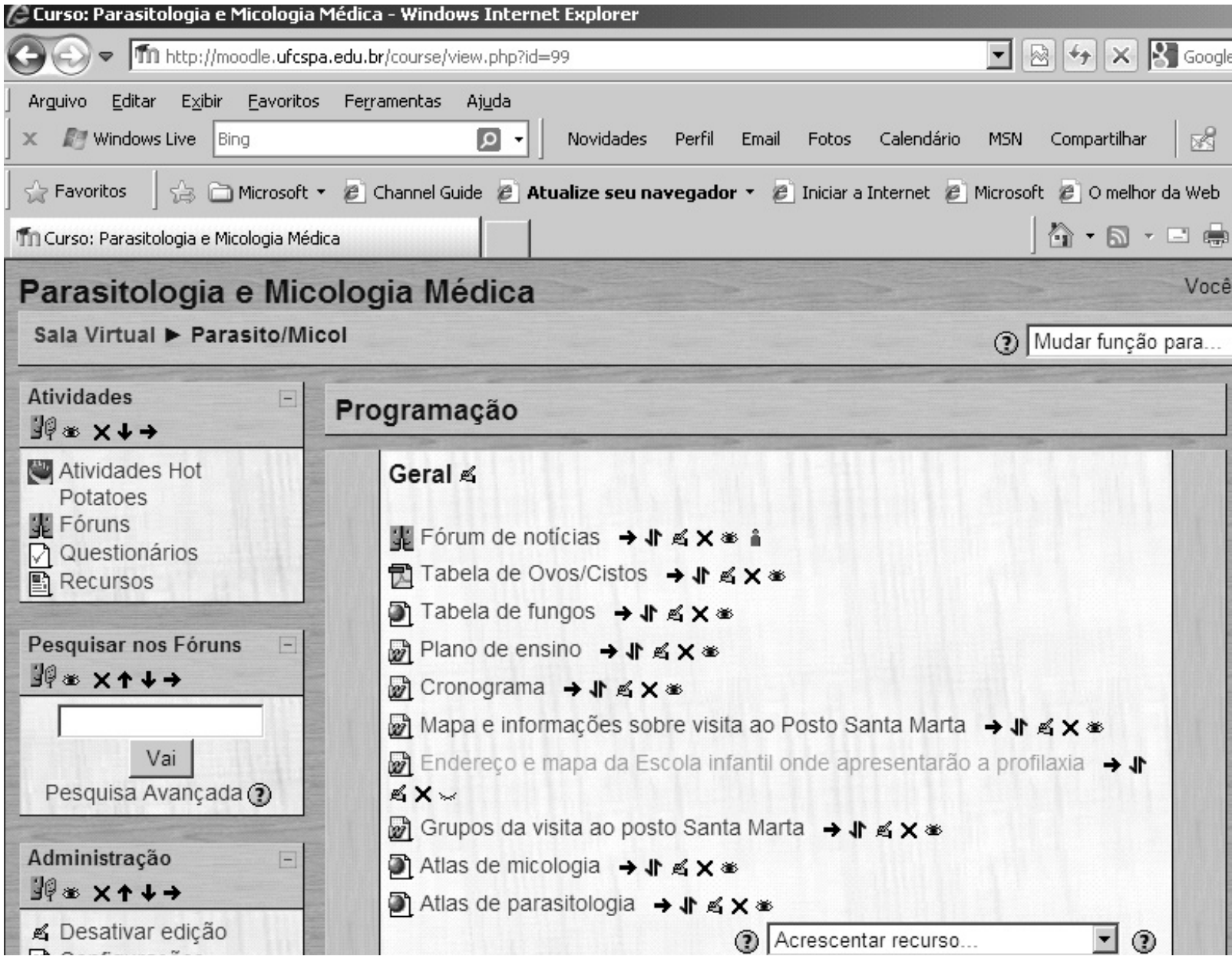


FIgURA 2

Atividades disponibilizadas para os alunos

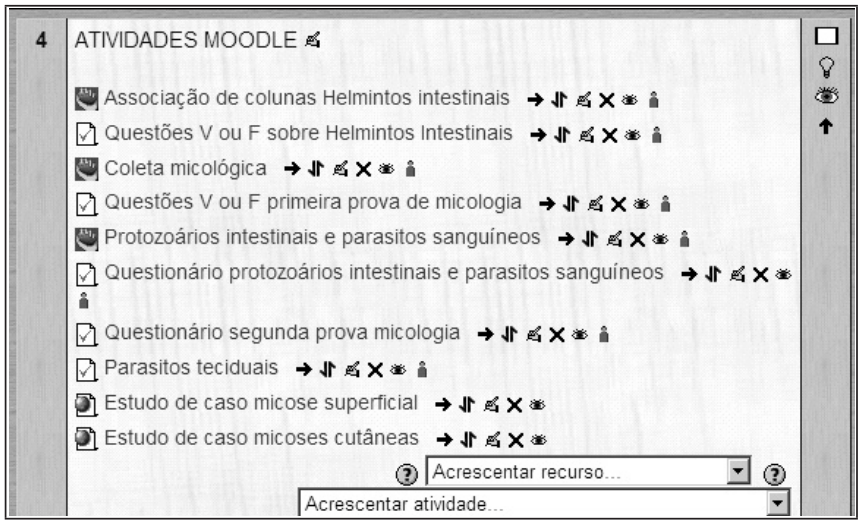

Todos os estudos de casos clínicos ou outras atividades são disponibilizados com tempo definido para a sua solução e antes da aula presencial, sendo os alunos informados por meio de mensagens, como apresentado na Figura 3. As mensagens e os acessos dos alunos ao site são registrados pela plataforma Moodle por meio do recurso elaboração de relatórios, visualizado em gráfico, como observado no Gráfico 1.
As respostas e os acessos são avaliados pelo professor e posteriormente discutidos juntamente com os conteúdos teóricos com o monitor tutor.

Os dados obtidos, comparados com as médias das notas dos alunos que participam ou não das atividades, são expressos como média \pm desvio padrão e são sujeitos ao teste t-Student. As diferenças entre os valores médios são consideradas significantes quando $P<0,05$

\section{DISCUSSÃO}

Lima e Linhares ${ }^{10}$ descrevem que a elaboração de bons problemas é fundamental em uma disciplina. O problema é uma ferramenta que o professor pode utilizar para motivar o aluno a resgatar conhecimentos arquivados na memória, instigar dúvidas científicas, integrar conhecimentos e dirigir o estudo. Nesta disciplina de Parasitologia e Micologia Médica, os problemas apresentados aos alunos são estudos de casos clínicos para culminar no diagnóstico clínico micológico ou parasitológico, além de outras atividades de fixação dos conteúdos.

Iglesias et al. ${ }^{11}$, em estudo semelhante, aplicaram o uso da metodologia de ensino presencial comparativamente com

Figura 3

Relatório de participação dos alunos através dos acessos

Earasito/Micol: Relatório de participação - Windows Internet Explorer

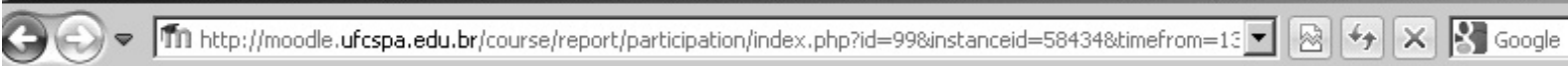

Arguivo Editar Exibir Eavoritos Ferramentas Ajụda

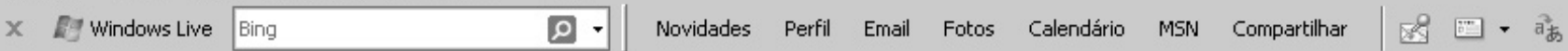

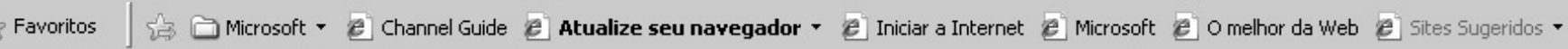

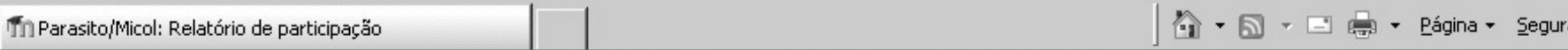

\section{Parasitologia e Micologia Médica}

Sala Virtual Parasito/Micol Relatórios Relatório de participação

Módulo de atividades Questionário segunda prova micologia

- Mostrar ações Mensagem

Olhar o verso 1 semanas $\mathbf{I}$

Questionário Acessos: view, view all, report

Questionário Mensagens: attempt, editquestions, review, submit

\section{Estudante}




\section{GRÁFICO 1}

Resultados dos acessos dos alunos na participação das atividades disponiblizadas

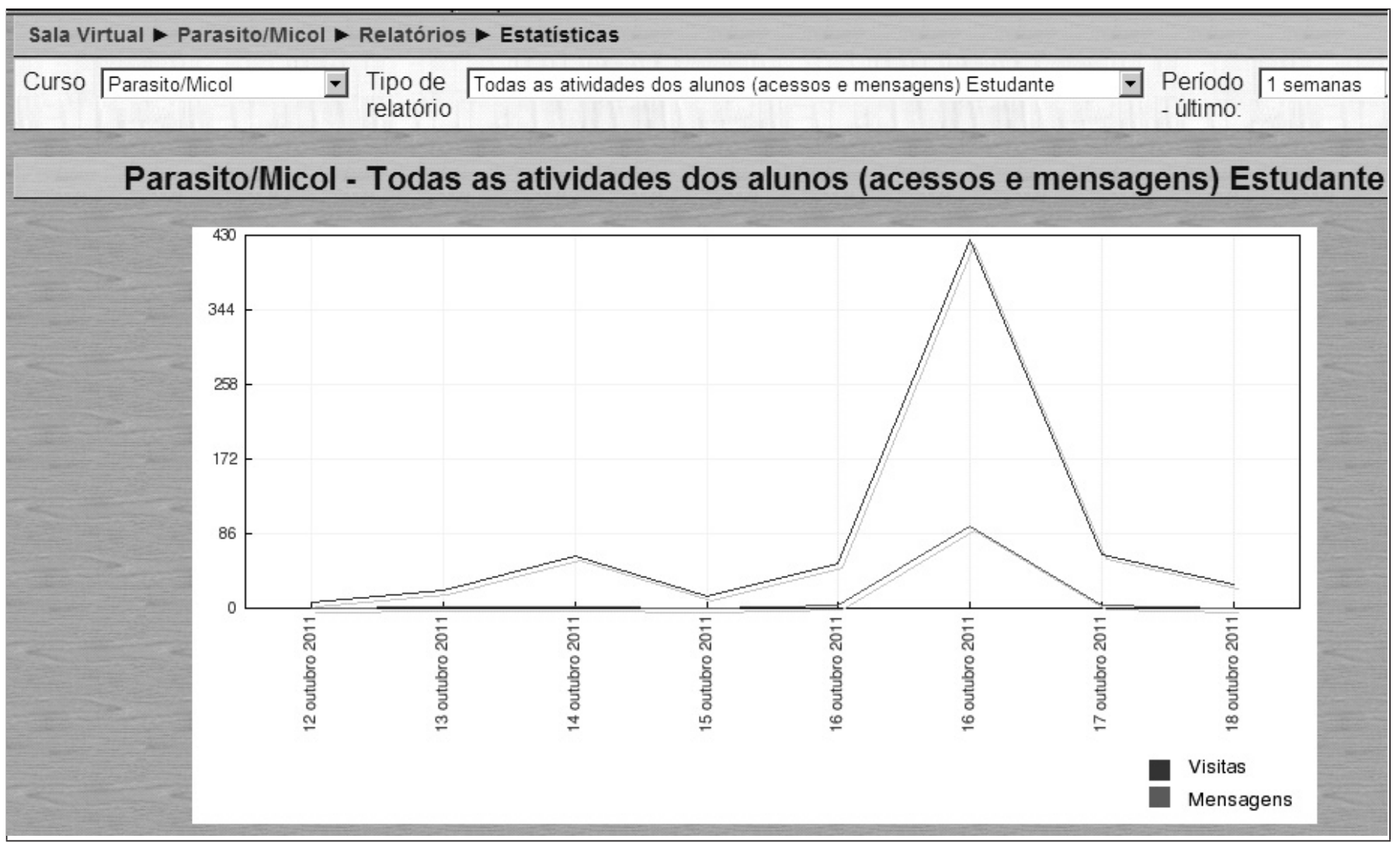

uma associação de ensino presencial, ensino com problemas de casos clínicos e trabalho em grupo para o estudo da Fisiologia e Anatomia na Universidade Europeia de Madri. Os pesquisadores concluíram que a metodologia de ensino mista permitiu que os alunos adquirissem habilidades e competências ao longo do período estudado, equilibrando as diferenças como parte do processo de aprendizagem. Fato semelhante pode ser observado no presente estudo, por intermédio da implantação desta complementação no ensino presencial da disciplina, e observado nas avaliações aplicadas, nas quais se verificou melhor desempenho dos alunos que participam das atividades a distância, quando comparados com os que não participam. Além disso, os alunos aprovaram sua utilização no ensino da disciplina, afirmando ser este método relevante em seu aprendizado.

Stahl et al. ${ }^{12}$ projetam sua perspectiva histórica relacionando a ciência e o aprendizado, assim como Vidal ${ }^{13}$ enfoca que a educação com reforço é um recurso importante para formar novos profissionais de modo efetivo e sem perda de qualidade do ensino oferecido. Esta metodologia mista, presencial e a distância, contribui positivamente para o aprendizado de forma dinâmica, reflexiva e autônoma. Os alunos podem estudar a qualquer hora, de qualquer lugar e em seu próprio ritmo, realizando as atividades em horários que lhes sejam mais favoráveis. Podem, também, esclarecer dúvidas e fazer comentários e contribuições a respeito das atividades que estão sendo realizadas. Uma avaliação contínua do aluno pela disciplina também complementa seu aprendizado.

Outro fato importante, relatado por Burnier et al. ${ }^{14}$, é que na aplicação de novas dinâmicas de ensino a experiência do professor, sua formação técnica e o acúmulo de experiências anteriores no processo da docência lhe possibilitam construir uma inserção na docência, melhorando, assim, o ensino e aprendizado do aluno. A experiência do docente neste processo de complementação no ensino presencial traz um reforço ao ensino e à aprendizagem. Além disso, o professor precisa estar atento e supervisionar todo o processo de aprendizagem de seus alunos, principalmente dos que demonstram mais dificuldades em aprender os conteúdos da disciplina. 
Botti e Rego ${ }^{15}$ reforçam que, mesmo com o uso de novas metodologias de ensino, o papel do professor é fundamental no envolvimento do aluno com o próprio aprendizado. O professor deve se subsidiar com informações completas sobre o desenvolvimento das atividades do aluno, acompanhando-o atentamente em sua trajetória. Nesse cenário, o monitor tutor é um guia e facilitador que auxilia o professor no processo da aprendizagem do aluno. Seguindo esse contexto, no presente estudo, a metodologia desenvolvida na disciplina de Parasitologia e Micologia Médica do curso de Medicina da UFCSPA se baseou no reforço do ensino presencial com uso da plataforma Moodle institucional.

\section{CONCLUSÕES}

O objetivo deste estudo - facilitar o aprendizado e a aquisição de conhecimentos pelos alunos - foi atingido. Os alunos podem utilizar os instrumentos de ensino em seu dia a dia, independentemente da presença do professor em sala de aula, e, inclusive, podem realizar as atividades em conjunto com os colegas e com a participação mútua entre eles.

A ferramenta mais utilizada foi o estudo de casos clínicos por meio do questionário. Os alunos entrevistados se manifestaram satisfeitos com o uso desta modalidade na disciplina.

$\mathrm{O}$ uso deste recurso de ensino na disciplina possibilitou abrir uma porta de inovação tecnológica na docência e na pesquisa, deixando em aberto a continuidade do processo de desenvolvimento de novas modalidades de ensino e pesquisa no campo da saúde.

Os resultados deste estudo apontam que a utilização de um complemento no ensino presencial de graduação nos cursos da área da saúde pode tornar mais efetiva a formação profissional. O emprego dessas ferramentas permitiu detectar um aumento significativo das notas dos alunos que participaram plenamente das atividades oferecidas. Esses dados apontam uma avaliação positiva do aprendizado dos estudantes que fizeram uso desse recurso.

Este estudo finalizou com a consciência dos docentes sobre as muitas opções disponíveis para qualificar o ensino presencial, sendo um desafio para eles buscar permanentemente estratégias significativas e interacionistas em suas atividades docentes.

\section{REFERÊNCIAS}

1. Hahn RU, Passerino L. Análise da Ação Pedagógica em AVAs: um estudo de caso no MOODLE.

Ciência em Movimento. 2011; 26:21-40.

2. Cyrino EG, Toralles-Pereira ML. Trabalhando com estratégias de ensino-aprendizado por descoberta na área da saú- de: a problematização e a aprendizagem baseada em problemas.Cad. Saúde Pública. 2004; 20(3):780-788.

3. Castanho ME. University level professors and their pedagogical practice in the healthcare área. Interface-Comunic Saúde Educ. 2002; 6(10):51-62.

4. Shen J, Hiltz SR, Bieber M. Learning Strategies in Online Collaborative Examinations. IEEE Transactions on Professional Communication. 2008; 51(Issue 1): 63-78.

5. Legoinha P, Pais J, Fernandes J. O Moodle e as comunidades virtuais de aprendizagem. Livro de Resumos do 7. Congresso Nacional de Geologia. Estremoz; 2006. v.3. p.841-844. Disponível em: http://www.dct.fct.unl.pt/ PLegoinha/CNGMood.pdf.

6. Cunha RM, Gross E, Santana LF, Sousa MCS. Motivar para o ensino à distância no ambiente moodle. Rev. da Pós-graduação. 2007; 1(2). Disponível em: http:/ / metodoead.blogspot.com/2009/01/motivar-para-o-ensino-distncia-no. html

7. Lampert JB. Dois séculos de escolas médicas no Brasil e a avaliação do ensino médico no Panorama atual e perspectivas. Gazeta Médica da Bahia. 2008; 78(supl. 1):31-37.

8. Trahasch S. From peer assessment towards collaborative learning. Proceedings of the $34^{\text {th }}$ Frontiers in Education. 2004; 2:5.

9. Varga CRR, Almeida VC, Germano CMR, Melo DG, Chadrá SGF, Souto BGA, Fontanella BJB, Lima VVL. Relato de experiência: o uso de simulações no processo de ensino-aprendizagem em medicina. Rev Bras Educ Méd. 2009; 33(2):84-95

10. Lima GZ, Linhares REC. Escrever bons problemas. Rev Bras Educ Méd.2008;32(2):197-201.

11. Iglesias GB, Berrade BI, Astray GMC. Nuevas metodologias docentes aplicadas al estúdio de la fisiologia y la anatomia: estúdio comparativo com el método tradicional. Rev Bras Educ Med. 2009;12(2):117-124.

12. Stahl G, Koschmann T, Suthers D. CSCL: An Historical Perspective. Based on a chapter. In: Sawyer RK, ed. Cambridge Handbook of the Learning Sciences. Cambridge, UK: Cambridge University Press; 2006.

13. Vidal E. Ensino a distância vs ensino tradicional. Porto: Universidade Fernando Pessoa; 2002. Disponível em: http:/ / www2.ufp.pt/ lmbg/monografias/evidal_mono.pdf.

14. Burnier S, Cruz RMR, Durães MN, Paz ML, Silva NA, Silva IMM. Histórias de vida de professores: o caso da educação profissional. Rev Bras Educ Bras. 2007;12(35).

15. Botti SHO, Rego STA. Preceptor, supervisor, tutor e mentor: quais são seus papéis? Rev Bras Educ Med. 2008; 32(3):363-373 


\section{CONTRIBUIÇÃO DOS AUTORES}

Adelina Mezzari, Isabele Iser, Adília Maria Pereira Wiebbelling e Liane Tarouco participaram da concepção inicial e acompanhamento do estudo, da coleta e análise dos dados, da concepção e elaboração da versão do artigo e da aprovação da versão encaminhada para publicação.

\section{CONFLITO DE INTERESSES}

Declarou não haver.

\section{ENDEREÇO PARA CORRESPONDÊNCIA}

Adelina Mezzari

Rua Sarmento Leite, 245

Bairro Centro - Porto Alegre

CEP 90050-170 — RS

E-mail: mezzari@ufcspa.edu.br 